\title{
Transcranial Brain Stimulation Techniques For Major Depression: Should We Extend TMS Lessons to tDCS?
}

\author{
Bernardo Dell'Osso* and A. Carlo Altamura
}

\author{
University of Milan, Milano (I), Dept of Neurosciences, Fondazione IRCCS Cà Granda, Ospedale Maggiore Policlinico, \\ Via F. Sforza 28, 20122, Milano (I), Italy
}

\begin{abstract}
Transcranial magnetic stimulation (TMS) and transcranial direct current stimulation (tDCS) are non-invasive brain stimulation techniques that, by means of magnetic fields and low intensity electrical current, respectively, aim to interefere with and modulate cortical excitability, at the level of dorsolateral prefrontal cortex, in patients with major depression and poor response to standard antidepressants. While the clinical efficacy of TMS in major depression has been extensively investigated over the last 10 years, tDCS has attracted research interest only in the last years, with fewer randomized clinical trials (RCTs) in the field. Nevertheless, in spite of the different rationale and mechanism of action of the two techniques, tDCS recent acquisitions, in relation to the treatment of major depression, seem to parallel those previously obtained with TMS, in terms of treatment duration to achieve optimal benefit and patient's history of drugresistance. After briefly introducing the two techniques, the article examines possible common pathways of clinical use for TMS and tDCS, emerging from recent RCTs and likely orienting future investigation with non invasive brain stimulation for the treatment of major depression.
\end{abstract}

Keywords: Brain stimulation, major depression, transcranial direct current stimulation (tDCS), transcranial magnetic stimulation (TMS).

Within transcranial brain stimulation techniques, both transcranial magnetic stimulation (TMS) and transcranial direct current stimulation (tDCS) represent non-invasive interventions, increasingly used in psychiatric research and clinical practice, to focally and selectively stimulate the dorso-lateral prefrontal cortex (DLPFC) in patients with major depression and poor response to standard antidepressant treatments.

While TMS utilizes coil-generated magnetic fields to penetrate the brain and, then, convert in electric current modulating cortical excitability, in tDCS, weak electric currents (1-2 mA) are directly applied to the scalp with the same purpose, by means of two electrodes: the anode (where the current enters), positioned over the left DLPFC, and the cathode (generally on the right DLPFC, where the current flows out).

While magnetic impulses can directly interfere with polarization process of cortical neurons and strengthen synaptic connections through mechanisms of long-term potentiation and depression, tDCS effects are supposed to be more oriented toward modulation of neurotransmitter systems and promotion of brain derived neurotrophic factor-dependent synaptic plasticity, even though tDCS induced changes in resting membrane potential, spontaneous neuronal firing rates, cerebral blood flow and metabolism as well as increase in myo-inositol brain content have been reported [1-3].

\footnotetext{
*Address correspondence to this author at the University of Milan, Milano (I), Dept of Neurosciences, Fondazione IRCCS Cà Granda, Ospedale Maggiore Policlinico, Via F. Sforza 28, 20122, Milano (I), Italy; Tel: 0039-02- 55035994; Fax: 0039-02- 50325994;

E-mail: bernardo.dellosso@unimi.it
}

In spite of a different rationale and peculiar mechanisms of action, both TMS and tDCS are aimed to restore the functional balance between the 2 brain hemispheres, which is supposed to be altered in major depression [4]. Current pathophysiological models, in fact, converge in suggesting that two major groups of brain regions - a "dorsal" and "ventral" network - account for the formation of the varied symptoms of depressive illness [4]. Within this theoretical framework, depression is hypothesized to involve concurrent hypoactivation of dorsal prefrontal regions and hyperactivation of ventral prefrontal regions, particularly in the left hemisphere [5]. Symptom remission is, therefore: supposed to require facilitation of hypoactive dorsal brain regions and inhibition of hyperactive ventral areas, which is actually what transcranial stimulation techniques aim to obtain $[4,5]$.

With respect to clinical use of transcranial stimulation techniques, compared to tDCS, repetitive TMS has been more extensively investigated in the field of major depression, over the last years, in terms of large randomized, sham controlled trials yelding meta-analyses [6], guidelines recommendations [7] as well as, in some countries, specific approval by local regulatory agencies. Taken as a whole, the aforementioned studies have allowed at least two important acquisitions, aimed to maximize treatment response and better characterize TMS candidate: 1) longer duration of treatment (i.e., more tms sessions) produce higher response and remission rates and 2) higher treatment resistance level to previous treatments reduces tms response. Therefore, even though other stimulation parameters still need to be further investigated (e.g., tms as monotherapy or augmentative treatment, need for and schedule of maintenance sessions after acute treatment, efficacy in unipolar vs bipolar depres- 
sion, etc.), it is currently well established that the best candidate for TMS response should be treated for not less than 3/4 weeks and should not be a highly treatment resistant subject.

With respect to tDCS, a more limited number of shamcontrolled studies has been published to date, yelding room for only two meta-analyses $[8,9]$, but no specific treatment guideline, in the field of major depression. In such perspective, however, the recently published, largest controlled trial (published after the aforementioned meta-analyses) showed that tDCS was superior to placebo/sham tDCS over a sixweek treatment course, as well as comparable efficacy to sertraline [10]. Of note, authors found that baseline severity and treatment resistance to more than 1 failed antidepressant trial were associated with a lower response. Previously, another sham-controlled, randomized trial found an increased number of responders after 6 weeks of treatment compared to 3 weeks, suggesting that antidepressant effects might be enhanced with longer treatment [11]. Taken as a whole, tDCS recent results in terms of duration of treatment and baseline level of treatment resistance, in relation to outcome, seem to parallel previous, large multicenter TMS studies [12, 13], supporting the perspective of a similar profile for candidate choice to transcranial non-invasive stimulation techniques. While further studies are required to confirm such aspects, the debate as to which technique to offer patients [14] should also start to consider more specifically tolerability-related issues and procedure-related costs as well as other clinical features that may orient clinician's choice.

\section{CONFLICT OF INTEREST}

The authors confirm that this article content has no conflict of interest.

\section{ACKNOWLEDGEMENTS}

Declared none.

\section{REFERENCES}

[1] Dell'Osso B, Priori A, Altamura AC. Efficacy and safety of transcranial direct current stimulation in major depression. Biol Psychiatry $2011 ; 69(8)$ : e23-4.
[2] Nitsche MA, Fricke K, Henschke U, et al. Pharmacological modulation of cortical excitability shifts induced by transcranial direct current stimulation in humans. J Physiol 2003; 553: 293-301.

[3] Fritsch B, Reis J, Martinowich K, et al. Direct current stimulation promotes BDNF-dependent synaptic plasticity: potential implications for motor learning. Neuron 2010; 66: 198-204.

[4] Mayberg HS. Limbic-cortical dysregulation: a proposed model of depression. J Neuropsychiatry Clin Neurosci 1997; 9: 471-81.

[5] Davidson RJ. Anterior electrophysiological asymmetries, emotion, and depression: conceptual and methodological conundrums. Psychophysiology 1998; 35: 607-14.

[6] Dell'Osso B, Camuri G, Castellano F, et al. Meta-review of metanalytic studies with repetitive transcranial magnetic stimulation (rTMS) for the treatment of major depression. Clin Pract Epidemiol Ment Health 2011; 7: 167-77.

[7] Rossi S, Hallett M, Rossini PM, Pascual-Leone A; Safety of TMS Consensus Group. Safety, ethical considerations, and application guidelines for the use of transcranial magnetic stimulation in clinical practice and research. Clin Neurophysiol 2009; 120(12): 200839.

[8] Berlim MT, Van den Eynde F, Daskalakis ZJ. Clinical utility of transcranial direct current stimulation (tDCS) for treating major depression: a systematic review and meta-analysis of randomized, double-blind and sham-controlled trials. J Psychiatr Res 2013; 47(1): $1-7$.

[9] Kalu UG, Sexton CE, Loo CK, Ebmeier KP. Transcranial direct current stimulation in the treatment of major depression: a metaanalysis. Psychol Med 2012; 42(9): 1791-800.

[10] Brunoni AR, Valiengo L, Baccaro A, et al. The sertraline vs. electrical current therapy for treating depression clinical study: results from a factorial, randomized, controlled trial. JAMA Psychiatry 2013; 70(4): 383-91.

[11] Loo CK, Alonzo A, Martin D, Mitchell PB, Galvez V, Sachdev P. Transcranial direct current stimulation for depression: 3-week, randomised, sham-controlled trial. Br J Psychiatry 2012; 200(1): 52-9.

[12] O'Reardon JP, Solvason HB, Janicak PG, et al. Efficacy and safety of transcranial magnetic stimulation in the acute treatment of major depression: a multisite randomized controlled trial. Biol Psychiatry 2007; 62(11): 1208-16.

[13] George MS, Lisanby SH, Avery D, et al. Daily left prefrontal transcranial magnetic stimulation therapy for major depressive disorder: a sham-controlled randomized trial. Arch Gen Psychiatry 2010; 67(5): 507-16.

[14] Priori A, Hallett M, Rothwell JC. Repetitive transcranial magnetic stimulation or transcranial direct current stimulation. Brain Stimul $2009 ; 2: 241-5$

Received: April 29, 2014

Revised: August 20,2014

Accepted: August 20,2014

(C) Dell'Osso and Altamura; Licensee Bentham Open.

This is an open access article licensed under the terms of the Creative Commons Attribution Non-Commercial License (http://creativecommons.org/licenses/by-nc/3.0/) which permits unrestricted, non-commercial use, distribution and reproduction in any medium, provided the work is properly cited. 